\title{
Preparation of Porous Hydroxyapatite/ $\alpha$-Tricalcium Phosphate Composites by a Colloidal Process
}

\author{
Hiroyuki Y. Yasuda ${ }^{1,2}$, Shigeo Mahara ${ }^{3}$, Naohiro Terashita ${ }^{1}$ and Yukichi Umakoshi ${ }^{1,2}$ \\ ${ }^{1}$ Department of Materials Science and Engineering and ${ }^{2}$ Frontier Research Center, Graduate School of Engineering, \\ Osaka University, Suita 565-0871, Japan \\ ${ }^{3}$ Sekisui Chemical Co. Ltd., 1, Koga, Shiga 528-8585, Japan
}

\begin{abstract}
Porous hydroxyapatite (HAp) $/ \alpha$-tricalcium phosphate $(\alpha$-TCP) composites were prepared by a colloidal process. HAp $/ \alpha$-TCP mixed powder was ultrasonically dispersed in an aqueous solution containing an optimum amount of polycarboxylic acid ammonium as polymer dispersant. After the colloidal process, polymethyl methacrylate (PMMA) was added to the slurries, followed by dehydration. A pressure filtration was found to be effective in avoiding the gravity segregation of PMMA. Numerous spherical pores with $100 \mu \mathrm{m}$ in mean diameter were introduced in the composites by mixing PMMA, which was burned out during sintering. The shape, size and volume fraction of pores could be controlled by those of PMMA added to the composites. The bending strength of HAp/ $\alpha$-TCP composites depended strongly on the porosity; the strength decreased exponentially with increasing the porosity. These composites may demonstrate higher solubility and faster bone growth than HAp alone.
\end{abstract}

(Received November 13, 2001; Accepted April 12, 2002)

Keywords: hydroxyapatite, biomaterials, microstructure, powder processing, colloidal process

\section{Introduction}

Enormous progress has been made in the research on biological tissue engineering for clinical use. ${ }^{1)}$ In tissue engineering, hydroxyapatite (HAp) has been widely used as a scaffold for bone regeneration and a carrier of bone morphogenetic proteins (BMP) because of its excellent biocompatibility. ${ }^{1)}$ However, HAp is too stable in vivo to be absorbed and substituted by regenerated new bone. It is well known that $\beta$ tricalcium phosphate $(\beta$-TCP) is more soluble in vivo. Since HAp and $\beta$-TCP behave as a seed of bone and a supplier of the ions, respectively, biphasic calcium phosphates $(\mathrm{CaP})$ composed of more stable HAp and more soluble $\beta$-TCP showed higher solubility and faster bone growth than HAp alone. ${ }^{2)}$ $\alpha$-TCP, which is stable above $1473 \mathrm{~K}$, dissolves in the body faster than both HAp and $\beta$-TCP. ${ }^{3)}$ Thus, the biocompatibility of HAp/ $\alpha$-TCP and HAp/ $\beta$-TCP composites are expected to be different from each other. However, there is little information on the preparation of HAp/ $\alpha$-TCP composites. In our previous paper, we prepared dense HAp $/ \alpha$-TCP composites by a colloidal process. ${ }^{4)}$ Spontaneous agglomeration of powder in a dry powder process due to van der Waals force leads to an inhomogeneous microstructure of sintered materials, resulting in poor mechanical properties. The colloidal process suppresses such agglomeration because of electrostatic, steric or electrosteric repulsive force by polymer dispersant. ${ }^{5-7)}$ An addition of appropriate amount of dispersant leads to a good dispersion of particles, resulting in good mechanical properties. ${ }^{5)}$ We succeeded in producing HAp $/ \alpha$-TCP composites with high relative density and homogeneous microstructure. ${ }^{4)}$ However, CaP ceramics should be porous materials containing $100 \mu \mathrm{m}$-sized pores to incorporate cells needed for bone regeneration and allow bone ingrowth. ${ }^{8)}$

A mixture of $\mathrm{CaP}$ powder with a polymeric powder followed by die-pressing may be the most widely used in producing porous $\mathrm{CaP}$ ceramics with controlled porosity. ${ }^{8)}$ The polymeric particles are burned out by heating and consequently numerous pores are formed in this ceramics. Nevertheless, the die-pressing leads to the plastic deformation of the polymeric powder, resulting in a shape change of the pores. In contrast, the colloidal process can disperse the polymeric powder and make it easy to obtain consolidated samples without severe pressing. ${ }^{5-7)}$ Therefore, porous CaP ceramics containing pores of controlled shape and size can be readily prepared by the colloidal process. Moreover, homogeneous microstructure obtained by the colloidal process may result in higher strength of $\mathrm{CaP}$ ceramics. In this paper, we prepared HAp/ $\alpha$-TCP composites by the colloidal process focusing on volume fraction of polymeric powder. A pressure filtration ${ }^{9)}$ was found to be effective in avoiding gravity segregation of HAp, $\alpha$-TCP and polymeric powder.

\section{Experimental Procedure}

$\alpha$-TCP and stoichiometric HAp (Taihei Chemical Industrial Co. $\alpha$-TCP-A and HAP-200) powders were mixed at a ratio of 40 to 60 by weight. Slurries were prepared with 30 vol\% mixed powder and 70 vol\% aqueous solution containing 4 mass $\%$ of polycarboxylic acid ammonium as an anion dispersant. ${ }^{4)}$ In the colloidal process, these slurries were ultrasonically dispersed for $20 \mathrm{~min}$, and then stirred by a magnetic agitator for $5 \mathrm{~h}$. The $\mathrm{pH}$ value of the slurries was adjusted to 10 by adding nitric or ammonium solution during the process. After the colloidal process, polymethyl methacrylate (PMMA) spherical particles $90 \mu \mathrm{m}$ in mean diameter were added to the slurries. The volume fraction $\left(V_{\mathrm{f}}\right)$ of PMMA powder to HAp $/ \alpha$-TCP mixed powder was selected from $10 \%$ to $60 \%$. After sufficient stirring for a few minutes, the slurries were quickly filtered through three types of filter at a uniaxial pressure of $4 \mathrm{MPa}$ to avoid gravity segregation of the HAp/ $\alpha$-TCP mixed powder and PMMA particles. This process is called pressure filtration, ${ }^{9)}$ and is schematically shown 


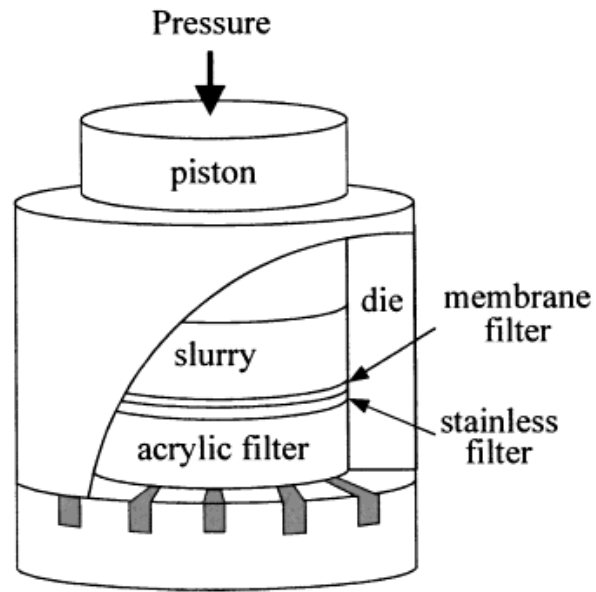

Fig. 1 A schematic illustration showing pressure filtration apparatus.

in Fig. 1. After the pressure filtration, the samples were sufficiently dried at $323 \mathrm{~K}$ for $24 \mathrm{~h}$. The consolidated samples were annealed at $773 \mathrm{~K}$ for $5 \mathrm{~h}$ to burn out PMMA powder and then sintered at $1473 \mathrm{~K}$ for $2 \mathrm{~h}$ at a heating rate of $200 \mathrm{Kh}^{-1}$. After sintering, these specimens were cooled at $100 \mathrm{Kh}^{-1}$. HAp-40 mass $\% \alpha$-TCP composites without PMMA powder were also sintered for $2 \mathrm{~h}$ in the temperature range from 1073 to $1473 \mathrm{~K}$ to compare with those prepared with PMMA.

The relative density of the sintered samples was measured by the Archimedes method. The microstructure of the sintered bodies was observed by a scanning electron microscope (SEM). A three-point bending test was performed on the basis of JIS R1601 using specimens with dimensions of $3 \mathrm{~mm}$ in thickness, $4 \mathrm{~mm}$ in width and $36 \mathrm{~mm}$ in length at a cross-head speed of $0.5 \mathrm{~mm} \mathrm{~min}^{-1}$ at room temperature in air. After the mechanical tests, these composites were immersed in the simulated body fluid (SBF) at $309.5 \mathrm{~K}$ for 3 days to check their biocompatibility.

\section{Results and Discussion}

Figure 2 shows SEM micrographs of porous HAp/ $\alpha$-TCP composites prepared by adding various amounts of PMMA particles during the colloidal process. At $V_{\mathrm{f}}=10.0 \%$, large spherical pores $100 \mu \mathrm{m}$ in diameter, which reflect the shape and size of the PMMA particles, are observed in Fig. 2(a). It is also noted that there are numerous $5 \mu \mathrm{m}$-sized pores in the composites. Further addition of PMMA particles results in an increase in the porosity and the connection of pores begins at $V_{\mathrm{f}}=33.8 \%$, as shown in Fig. 2(b). The $100 \mu \mathrm{m}$-sized pores frequently connect with each other forming a successive pore structure at $V_{\mathrm{f}}=56.1 \%$, while the density of small pores seems to decrease (Fig. 2(c)). Although $\mathrm{CO}_{2}$ gas formation caused by burned out PMMA sometimes leads to fracture of porous materials, the interconnection of pores provides an exit for the gas, resulting in highly porous composites without fracture. Thus, porous HAp $/ \alpha$-TCP composites produced using PMMA demonstrate uniform size, spherical shape and homogeneous distribution of pores at any $V_{\mathrm{f}}$. Moreover, the fast dissolution of the $\alpha$-TCP phase ${ }^{10)}$ may assist the pore continuity, which is desirable for introducing cells needed for bone regeneration. In addition, the pressure filtration used in this study is attributed to the suppression of the gravity segregation of HAp $/ \alpha$-TCP powder and PMMA, resulting in the homogeneous microstructure. In a high magnification image of the specimen at $V_{\mathrm{f}}=10.0 \%$, small amount of $\alpha$-TCP is transformed to calcium-deficient hydroxyapatite (DAp) by hydrolysis (Fig. 2(d)), since the temperature of the slurries exceeded $353 \mathrm{~K}$ due to ultrasonic dispersion. ${ }^{11)}$ But the volume fraction of DAp seems to be too small to influence the solubility of the specimen. Yuan et al. reported that porous $\alpha$-TCP implanted to dogs exhibit fast dissolution, but is not hydrated to DAp. ${ }^{12)}$ Therefore, the high solubility of $\alpha$-TCP may assist new bone formation on HAp.

Figure 3 is a SEM micrograph of HAp/ $\alpha$-TCP composites sintered at $1273 \mathrm{~K}$ for $2 \mathrm{~h}$ without PMMA. Although the porosity of the specimen is as much as $46.0 \%$, the formation of large pores of more than $100 \mu \mathrm{m}$ cannot be confirmed; only numerous small pores of less than $10 \mu \mathrm{m}$ exist in the specimen. Moreover, $\alpha$-TCP was transformed to $\beta$-TCP at $1273 \mathrm{~K}$. Therefore, HAp/ $\alpha$-TCP composites with $100 \mu \mathrm{m}$-sized pores cannot be produced solely by controlling the sintering temperature and without PMMA particles.

Figure 4 shows the relationship between porosity of $\mathrm{HAp} / \alpha$-TCP composites and volume fraction of PMMA powder. HAp-40 mass $\% \alpha$-TCP composites sintered at $1473 \mathrm{~K}$ for $2 \mathrm{~h}$ have $23.4 \%$ porosity even without PMMA. The porosity of the composites increases linearly with an increase in $V_{\mathrm{f}}$ to $20 \%$. In the region, the measured porosity of the composites is consistent with the ideal value estimated under the assumption that the increment of porosity is equal to the volume fraction of PMMA particles. In contrast, addition of PMMA more than $20 \%$ results in downward deviation of the measured porosity from the ideal one. At higher content of PMMA, numerous large pores corresponding to PMMA particles behave as a sink site for small pores resulting in the negative deviation of the porosity. Thus, the porosity of HAp/ $\alpha$ TCP composites can be readily controlled by changing the volume fraction of PMMA particles.

Figure 5 shows variation in the bending strength of HAp $/ \alpha$ TCP composites prepared with or without PMMA as a function of porosity. The previous result of the bending strength of sintered HAp is also plotted in the figure. ${ }^{13)}$ The bending strength $(\sigma)$ and the porosity $(P)$ of both HAp/ $\alpha$-TCP composites and HAp satisfy the following equation: ${ }^{14)}$

$$
\sigma=\sigma_{0} \exp (-b P)
$$

where $b$ is constant and $\sigma_{0}$ is the bending strength without pores. The $\sigma_{0}$ of HAp $/ \alpha$-TCP composites and HAp are $228 \mathrm{MPa}$ and $112 \mathrm{MPa}$, respectively. The change in bending strength of HAp/ $\alpha$-TCP composites prepared using PMMA particles with porosity is almost the same as that produced by the control of sintering temperature. Moreover, the scatter of the bending strength of the composites at each condition was confirmed to be minimal. Although there are many $100 \mu \mathrm{m}-$ sized pores in the composites prepared using PMMA, the homogeneous distribution of the pores leads to inhibition of the decrease and the scattering in the bending strength. Moreover, small pores less than $10 \mu \mathrm{m}$ were absorbed by $100 \mu \mathrm{m}$-sized pores at higher PMMA contents, as shown in Fig. 2. This suppresses a linkage of pores on crack propagation, resulting in an increase in the bending strength. The bending strength of 


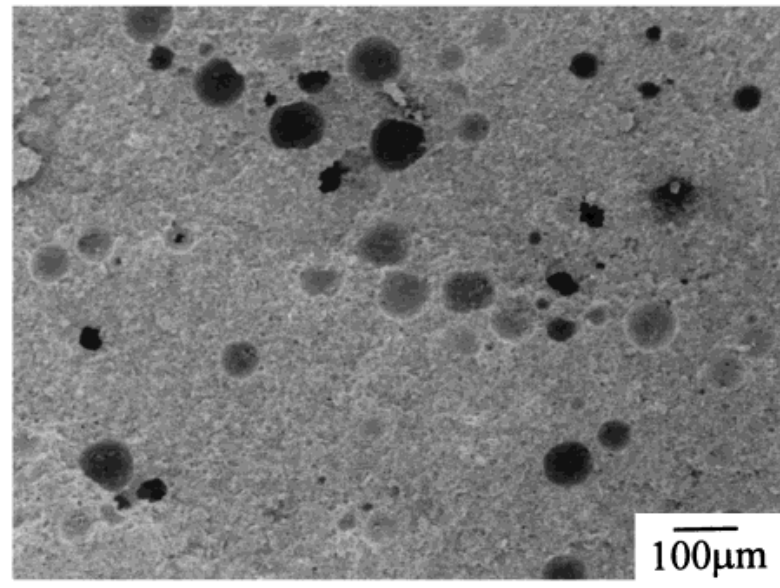

(a)

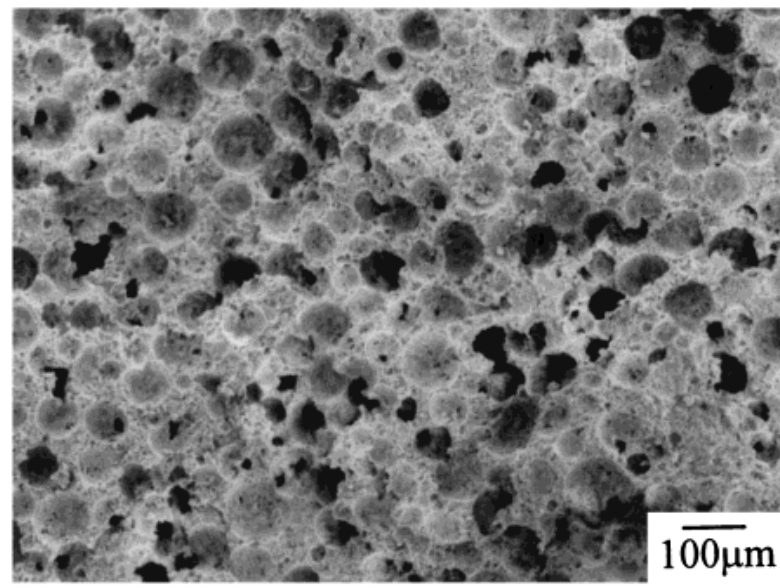

(c)

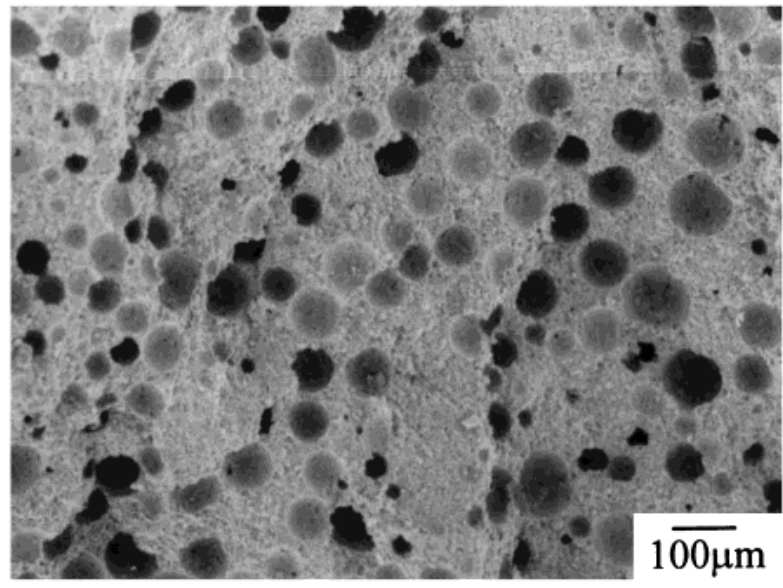

(b)

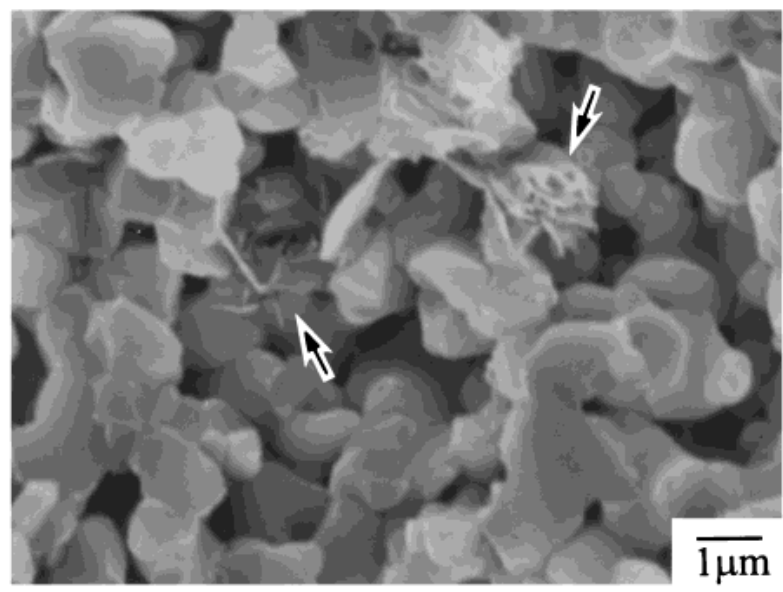

(d)

Fig. 2 SEM micrographs of porous HAp/ $\alpha$-TCP composites prepared by adding various amount of PMMA particles; (a) 10.0 vol\%, (b) $33.8 \mathrm{vol} \%$, and (c) $56.1 \mathrm{vol} \%$. (d) a high magnification image at $10.0 \mathrm{vol} \%$. All specimens were sintered at $1473 \mathrm{~K}$ for $2 \mathrm{~h}$. Arrows indicate DAp.

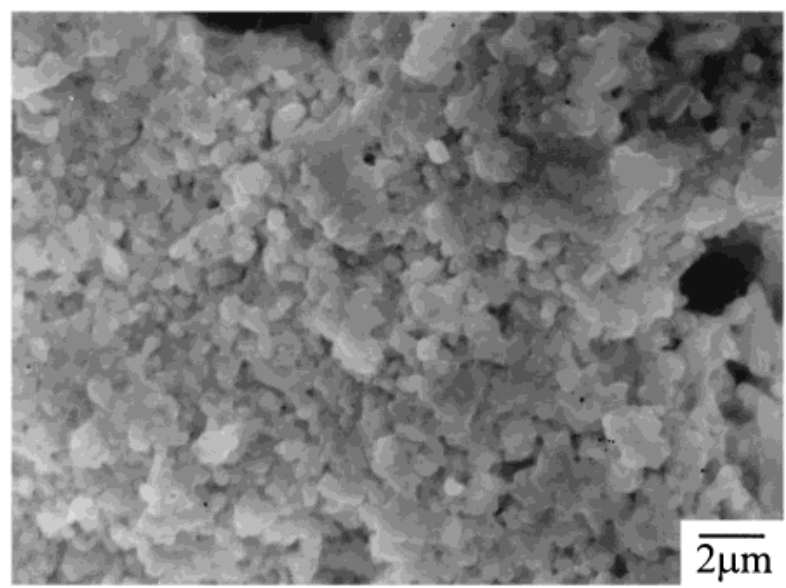

Fig. 3 A SEM micrograph of porous HAp/ $\alpha$-TCP composites sintered at $1273 \mathrm{~K}$ for $2 \mathrm{~h}$.

HAp/ $\alpha$ TCP composites is higher than that of HAp alone in the lower porosity region of less than $30 \%$, while it is smaller in the higher region. Since the optimum porosity of $\mathrm{CaP}$ ceramics was reported to be about $40 \%, \mathrm{HAp} / \alpha$-TCP composites

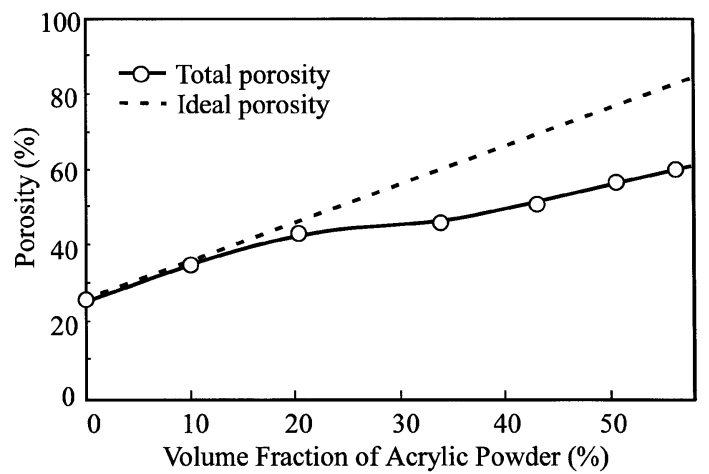

Fig. 4 Change in total porosity of HAp $/ \alpha$-TCP composites with volume fraction of PMMA particles. Ideal porosity estimated from the volume fraction of PMMA is also plotted.

demonstrate lower bending strength than HAp at optimum porosity. However, the high solubility and fast bone growth of HAp $/ \alpha$-TCP composites in vivo make them extremely suitable for clinical use.

Figure 6 shows a SEM micrograph of HAp/ $\alpha$-TCP composites immersed in SBF solution for 3 days. In the com- 


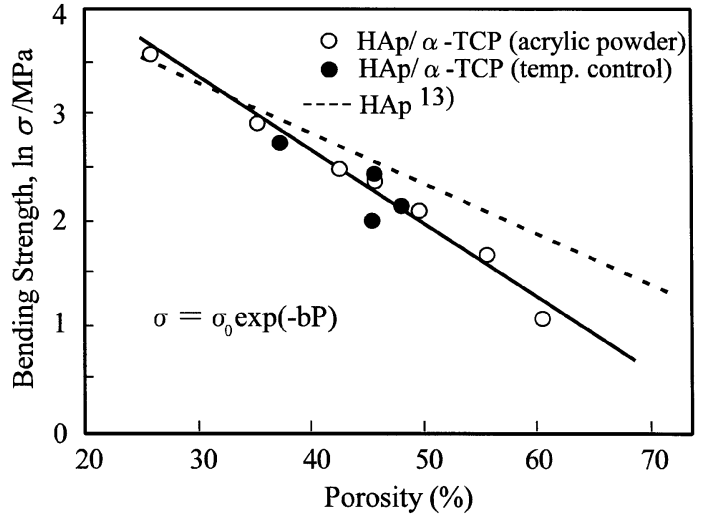

Fig. 5 Variation in bending strength of HAp and HAp/ $\alpha$-TCP composites with total porosity; $(\bigcirc)$ sintered at $1473 \mathrm{~K}$ for $2 \mathrm{~h}$ with various amount of PMMA particles, $(\mathbf{O})$ sintered at various temperatures, (-- ) HAp. ${ }^{13)}$

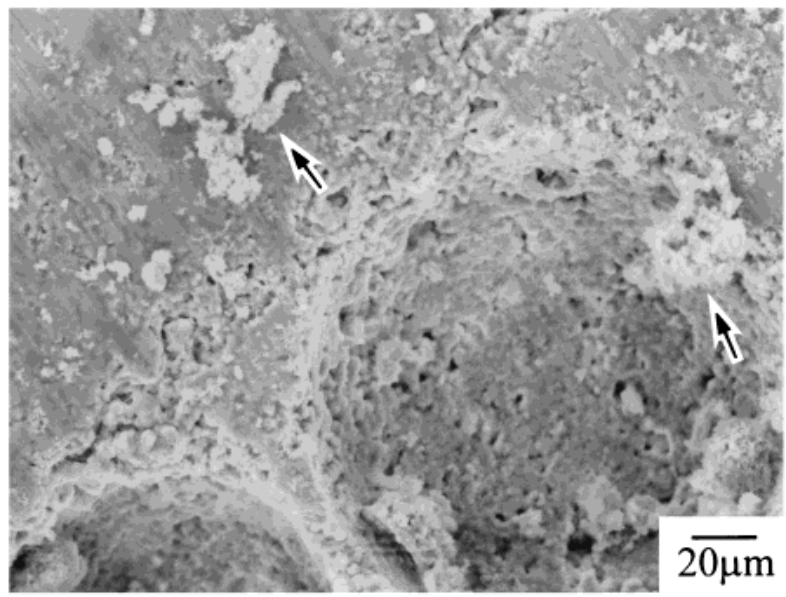

Fig. 6 A SEM micrograph of HAp $/ \alpha$-TCP composites at $V_{\mathrm{f}}=33.8 \%$. The specimen was immersed in the SBF at $309.5 \mathrm{~K}$ for 3 days. Arrows indicate examples of nucleated apatite.

posites at $V_{\mathrm{f}}=33.8 \%$, white apatite crystals are observed to nucleate on the surface of composites, although the density is not so high. Since these composites were sintered at $1473 \mathrm{~K}$, the zeta potential of HAp is known to be positive, ${ }^{15)}$ resulting in low ability of bone formation. Sintering at low temperatures below $1273 \mathrm{~K}$ is effective in the nucleation of new bone. However, $\alpha$-TCP is not stable below $1273 \mathrm{~K}$. Some modifications should be still needed to accelerate bone formation.

\section{Conclusions}

Porous HAp $/ \alpha$-TCP composites were prepared by the colloidal process and the porosity was controlled by adding PMMA particles. The following conclusions were reached:

(1) Porous HAp $/ \alpha$-TCP composites can be prepared by adding selected amounts of PMMA particles in the course of the colloidal process. The size, shape and distribution of pores can be easily controlled in this process. In particular, large pores $100 \mu \mathrm{m}$ in diameter are easy to form, while the control of sintering temperature is not effective for the introduction of large pores suitable for bone regeneration. Moreover, a pressure filtration is effective in suppressing gravity segregation of HAp, $\alpha$-TCP and PMMA particles.

(2) The porosity of HAp/ $\alpha$-TCP composites depends strongly on the volume fraction of PMMA powder. HAp/ $\alpha$ TCP composites with controlled porosity can be readily prepared using the correlation curve between the porosity of the composites and the volume fraction of PMMA powder.

(3) The bending strength $(\sigma)$ and the porosity $(P)$ of HAp $/ \alpha$-TCP composites satisfy the following equation: $\sigma=$ $\sigma_{0} \exp (-b P)$. In spite of large pores in the composites prepared with PMMA particles, the homogeneous pore distribution prevents a decrease and scattering of the bending strength.

\section{Acknowledgements}

This work was supported by the Research for the Future Program of the Japan Society for the Promotion of Science (JSPS) (Project: Biological Tissue Engineering) (JSPSRFTF98I00201) and a Grant-in-Aid for Scientific Research and Development from the Japanese Ministry of Education, Culture, Sports, Science and Technology.

\section{REFERENCES}

1) M. J. Yaszemski, R. G. Payne, W. C. Hayes, R. Langer and A. G. Mikos: Biomaterials 17 (1996) 175-185.

2) G. Daculsi: Biomaterials 19 (1998) 1473-1478.

3) H. Aoki: Medical Applications of Hydroxyapatite, Ishiyaku Euro America, St. Louis, (1994) 13-74.

4) H. Y. Yasuda, S. Mahara, T. Nishiyama and Y. Umakoshi: Sci. Tech. Adv. Mater. 3 (2002) 29-33.

5) H. Y. Yasuda, S. Mahara, Y. Umakoshi, S. Imazato and S. Ebisu: Biomaterials 21 (2000) 2045-2049.

6) I. A. Aksay, F. F. Lange and B. I. Davis: J. Am. Ceram. Soc. 66C (1983) 190-192.

7) J. Cesarano III, I. A. Aksay and A. Bleier: J. Am. Ceram. Soc. 71 (1988) 250-255.

8) D. M. Liu: Key Eng. Mater. 115 (1996) 209-232.

9) F. F. Lange and K. Y. Miller: Am. Ceram. Soc. Bul. 66 (1987) 14981504.

10) T. Nakano, K. Kaibara, Y. Umakoshi, S. Imazato and S. Ebisu: Boundary, 16 (2000) 20-22.

11) H. Monma, S. Ueno and T. Kanazawa: J. Chem. Tech. Biotechnol. 31 (1981) 15-24.

12) H. Yuan, J. D. de Bruijn, Y. Li, J. Feng, Z. Yang, K. de Groot and X. Zhang: J. Mater. Sci. Mater. in Med. 12 (2001) 7-13.

13) T. Nakajima: Bul. Ceram. Soc. Jpn. 34 (1999) 516-521.

14) R. W. Davidge and A. G. Evans: Mater. Sci. Eng. 6 (1970) 281-286.

15) S. Tamba: Journal of joint surgery 8 (1989) 65-71. 